\title{
PREJUICIO SUTIL Y ACTITUDES HACIA LA INCLUSIÓN DEL PROFESORADO Y ALUMNADO MIGRANTE MAGREBI
}

\author{
Miguel Ángel Albalá Genol \\ Universidad Autónoma de Madrid. Dpto. Interfacultativo de Psicología Evolutiva y de la Educación. \\ Facultad de Formación del Profesorado y Educación. \\ miguel.albala@uam.es \\ Juan Ignacio Guido \\ Universidad de Buenos Aires \\ juanguido@uba.psi.ar \\ Joaquín Ungaretti \\ Universidad de Buenos Aires, Consejo Nacional de Investigaciones Científicas y Técnicas (CONICET) \\ juanguido@uba.psi.ar \\ Antonio Francisco Maldonado Rico \\ Universidad Autónoma de Madrid. Dpto. Interfacultativo de Psicología Evolutiva y de la Educación. \\ Facultad de Formación del Profesorado y Educación. \\ antonio.maldonado@uam.es
}

Recepción Artículo: 18 mayo 2021 Admisión Evaluación: 18 mayo 2021 Informe Evaluador 1: 22 mayo 2021 Informe Evaluador 2: 27 mayo 2021 Aprobación Publicación: 02 junio 2021

\section{RESUMEN}

En las últimas décadas, muchas sociedades han experimentado un proceso de transformación, siendo la amplia diversidad sociocultural uno de los principales rasgos de una ciudadanía cada vez más globalizada. En este sentido, numerosas investigaciones se han centrado en estudiar las actitudes hacia la inclusión de la población migrante en diferentes contextos. Sin embargo, no han sido tantos los esfuerzos para analizar dichas actitudes en el ámbito educativo, ni en indagar otras posibles variables que puedan relacionarse con éstas. Desde una perspectiva inclusiva, los sistemas educativos se constituyen como uno de los principales instrumentos culturales para poder mejorar la integración social y la convivencia intercultural en las sociedades. Además, algunos antecedentes sugieren que diferentes formas de prejuicio, ya sean sutiles o manifiestas, se relacionan con las actitudes de la ciudadanía hacia cuestiones como la inclusión educativa del alumnado migrante. En el presente estudio participaron 481 personas adultas españolas (52\% mujeres y $48 \%$ hombres) con edades comprendidas entre los 18 y los 88 años, que cumplimentaron un cuestionario autoadministrable sobre actitudes hacia inclusión educativa, los niveles de prejuicio hacia personas migrantes magrebíes y variables socio-demográficas. Los resultados evidenciaron que las mujeres muestran actitudes significativamente más favorables hacia la inclusión 


\section{PREJUICIO SUTIL Y ACTITUDES HACIA LA INCLUSIÓN DEL PROFESORADO Y ALUMNADO MIGRANTE MAGREBÍ}

educativa del alumnado y profesorado migrante, en comparación con los hombres. Además, se hallaron relaciones negativas entre el prejuicio sutil hacia personas migrantes magrebíes y las actitudes favorables que poseen hacia la inclusión de alumnado y profesorado de dicho origen en las instituciones educativas. Finalmente, se discute la implicación de las variables estudiadas para contar en el futuro con instituciones y comunidades educativas más inclusivas, socialmente justas e interculturales.

Palabras clave: inclusión educativa; interculturalidad; prejuicio; profesorado; alumnado

\section{ABSTRACT}

Subtle prejudice and attitudes towards inclusion of maghrebi migrant teachers and students. In the last decades, many societies have undergone a process of transformation, with a great sociocultural diversity being increasingly frequent among citizens. In this sense, numerous researches have focused on studying attitudes towards the inclusion of the migrant population in different contexts. However, there have not been so many efforts to analyze these attitudes in the educational field, or to study other possible variables that may be related to them. Education is known as one of the main mechanisms, from an inclusive perspective, by which is able to improve social integration and intercultural coexistence in societies. In addition, previous studies suggest that different forms of prejudice, whether subtle or blatant, are related to citizens' attitudes towards issues such as the educational inclusion of migrant students. The sample of the study consisted of 481 Spanish students (52\% women and 48\% men) aged between 18 and 88 years, who completed a self-administrated questionnaire which inquired attitudes towards educational inclusion, levels of prejudice towards Maghreb migrants and socio-demographic variables. The results demonstrated that women show significantly more favorable attitudes towards the educational inclusion of migrant students and teachers, compared to men. In addition, negative correlations were found between the subtle prejudice towards Maghreb migrants and the favorable attitudes they have towards the inclusion of students and teachers of that ethnical population in educational institutions. Finally, the implication of the variables studied to have in the future more inclusive, socially just and intercultural institutions and communities is discussed.

Keywords: educational inclusion; interculturality; prejudice; professorate; students

\section{INTRODUCCIÓN}

En las últimas décadas se han incrementado exponencialmente los movimientos y flujos migratorios en todo el mundo. En este sentido, uno de los mayores retos de las sociedades modernas, tanto en el presente como en el futuro, corresponde al proceso de integración y convivencia intercultural entre los grupos cada vez más culturalmente diversos. Así, si bien a nivel mundial los gobiernos y los sistemas educativos han desarrollado estrategias sociales para mejorar dichos procesos, hasta ahora ninguna ha demostrado ser exitosa en todos los contextos (Civalero et al., 2019). De esta forma, el prejuicio, en cualquiera de sus manifestaciones, tiene su origen en la manera de interacción entre grupos mayoritarios y asociados a la cultura dominante que no reconocen a otro grupo minoritario (Licata et al. 2011). Con ello, pese a que en países como España actualmente los derechos formales de la ciudadanía migrante se encuentran parcialmente reconocidos, en tanto continúen reproduciéndose determinados prejuicios, su inclusión en las sociedades y la convivencia intercultural seguirán siendo horizontes lejanos de alcanzar.

Siguiendo a Rueda y Navas (1996), podemos señalar que en la convivencia democrática existe una amplia creencia hacia los ideales justos e igualitarios, que va vinculada al rechazo hacia expresiones abiertas de comportamientos discriminatorios en función de elementos identitarios, tales como la raza, la religión o la etnia. Sin embargo, en los últimos años se observan formas implícitas y explícitas de discriminación sobre la población migrante, en múltiples contextos como es el socio-educativo. En España, particularmente en los últimos tiempos han circulado discursos de odio con el objetivo potenciar la expresión de enunciados xenófobos contra ciertas minorías provenientes de otros países (Bustos et al., 2019). El colectivo de personas migrantes magrebíes es uno de los más afectados por las diversas formas de discriminación y racismo, derivadas de los fuertes prejuicios 
establecidos. La cercanía geográfica y los vínculos culturales entre España y algunos de los países que conforman el Magreb, hace que sea uno de los grupos extranjeros más presentes desde la última década del pasado siglo. En este sentido, según el Instituto Nacional de Estadística - INE (2020) en el año 2020 se encontraban conviviendo en España como grupos mayoritarios de origen magrebí: personas de origen marroquí (865.945); de origen argelino (66.893); de origen mauritano (8.392); y de origen tunecino (2.788). Concretamente en el contexto educativo, en términos generales se ha pasado de una escolarización de 107.301 alumnas y alumnos migrantes en el curso académico 1999/2000 a ascender dicha cifra hasta 863.952 estudiantes en el curso 2020/2021 (MECD, 2021). Por tanto, es evidente que en el contexto educativo existe una mayor diversidad sociocultural en la actualidad, que requiere de una presencia cada vez mayor de equipos de profesionales educativos con una perspectiva intercultural. Se hace necesario analizar de forma detallada la representación de los diferentes colectivos de alumnado migrante existentes en el contexto educativo. Dicho análisis se plantea como fundamental para lograr uno de los objetivos básicos relacionados con la inclusión educativa: la búsqueda de la justicia social (Sapon-Shevin, 2013). Desde esta perspectiva, uno de los retos para la búsqueda de un sistema educativo más justo es lograr un mayor reconocimiento y representación de todos los grupos socioculturalmente diversos, cada vez más presentes en las comunidades educativas.

El prejuicio por razones étnicas y culturales se vale de aspectos particulares de las minorías, connotándolos negativamente y utilizándolos como creencias legitimadoras de las prácticas discriminatorias (Chambers et al., 2013). Si bien dicho prejuicio ha sido foco de análisis en múltiples contextos sociopolíticos, en otros ámbitos tan institucionalizados como el educativo, no se les ha prestado tanta atención. Desde esta perspectiva, el prejuicio tradicionalmente se estudiaba empíricamente por medio del análisis de las formas más manifiestas del fenómeno, captando formas extremas e intolerantes de racismo (Rueda \& Navas, 1996), pasando desapercibidas diversas formas de prejuicio sutiles y más presentes dentro diferentes ámbitos como el educativo. En este sentido, los instrumentos de evaluación desarrollados en relación al prejuicio manifiesto comenzaron a manifestar evidencia de niveles cada vez menores de prejuicio, aunque seguían registrándose desigualdades sociales y prácticas discriminatorias (Gaertner y Dovidio, 1986). Así, estas formas manifiestas de prejuicio (relacionadas actitudes explícitas racistas y xenófobas) en instituciones como la educativa cada vez son menos permitidas. Sin embargo, continúan existiendo otras formas sutiles de prejuicio y actitudes desfavorables hacia la inclusión de los agentes educativos de origen migrante.

Según autores como Pettigrew y Meertens (1995), las evaluaciones de prejuicio exclusivamente manifiesto muestran incapacidad para captar otras novedosas modalidades de discriminación que seguían manifestando los grupos socioculturales diversos, desarrollaron una escala sensible a expresiones de prejuicio también sutiles, como una herramienta válida para contrastar la misma problemática. Además, dicho prejuicio sutil se encuentra relacionado con diferentes actitudes y comportamientos que las personas desarrollan hacia un grupo social concreto y son normativamente aceptadas en las sociedades occidentales. Entre los componentes definidos en relación al prejuicio sutil, se encuentran (Pettigrew y Meertens,1995): una primera dimensión que implicaría la defensa de valores tradicionales propios del endogrupo, ya que se presupone que los miembros del exogrupo (profesorado y alumnado migrante magrebí) actúan de maneras impropias; en segundo lugar, dicha manifestación sutil del prejuicio entrañaría la exageración de las diferencias culturales, conllevando estereotipos, y siendo éste el motivo que justifica la situación desfavorecida del exogrupo; por último, también conlleva la negación encubierta de respuestas emocionales positivas y actitudes favorables hacia la inclusión del exogrupo.

En base a los tres componentes del prejuicio sutil citados (Pettigrew y Meertens, 1995) y a los hallazgos de diversos estudios (Albalá et al., 2021; Arroyo, 2013) este se relaciona con las actitudes y creencias que las personas poseen acerca de la necesidad y pertinencia de buscar la inclusión socioeducativa del alumnado y profesorado migrante. De esta manera, si se aspira a lograr una convivencia intercultural de reconocimiento y respeto cultural, a corto y medio plazo se plantea como uno de los mayores retos el abordaje desde el marco educativo, con y para comunidades educativas cada vez más diversas. Por ello, en los próximos años uno de los grandes 
objetivos debe centrarse en el análisis acerca de los diferentes factores formativos y psicosociales que influyen en el desarrollo de prácticas inclusivas, que reduzcan los prejuicios y promuevan dinámicas de convivencia intercultural.

\section{OBJETIVOS DE LA INVESTIGACIÓN}

Los objetivos de la presente investigación fueron, en primer lugar, analizar las actitudes hacia la inclusión educativa del alumnado y profesorado migrante magrebí que tiene la población española, para en segundo lugar, indagar las relaciones que existen entre dichas actitudes y los niveles de prejuicio sutil hacia el profesorado y alumnado migrante magrebí.

\section{MUESTRA Y/O PARTICIPANTES}

En el estudio participaron 481 personas adultas residentes de diferentes comunidades autónomas de España, con una edad comprendida entre los 18 y los 88 años ( $M=53,1 ; D T=13,85)$, siendo el $52 \%$ de género femenino ( $n=251)$ y el $48 \%$ de género masculino ( $n=230$ ). Las personas que participaron del estudio lo hicieron de manera anónima y se le informó que los resultados serían utilizados únicamente con fines académicos.

\section{METODOLOGÍA Y/O INSTRUMENTOS UTILIZADOS}

Los datos han sido recolectados a partir de 2 instrumentos de evaluación de índole autoadministrable que indagaban las siguientes variables y escalas:

Prejuicio Sutil: se utilizó una adaptación al contexto español (Rueda \& Navas, 1996), de la escala original desarrollada por Pettigrew y Meertens (1995). La misma consta de 10 items con un formato de respuesta de tipo Likert con 5 anclajes, donde $1=$ Totalmente en desacuerdo, 2= Algo en desacuerdo, $3=$ Ni de acuerdo ni en desacuerdo, 4= Algo de acuerdo y $5=$ Totalmente de acuerdo.

Actitudes hacia la interculturalidad en el ámbito educativo: se utilizaron 5 items diseñados y testeados en estudios previos (Albalá et al., 2021) que permiten dar cuenta de las actitudes que tienen las personas hacia inclusión de los colectivos migrantes al ámbito educativo, teniendo en cuenta las actitudes tanto hacia el profesorado como al alumnado migrante magrebí. Para ello, se realizó la adaptación de los ítems hacia el colectivo migrante magrebí en particular. La temática evaluada se relaciona con las actitudes hacia políticas públicas que favorezcan la inclusión del colectivo magrebí en el ámbito escolar (por ejemplo: "el gobierno debe garantizar que los/as hijos/as de inmigrantes magrebíes reciban la educación necesaria") y universitario (por ejemplo: "las universidades deberían garantizar el acceso de estudiantes inmigrantes magrebíes"). El formato de respuesta es de tipo Likert con 5 anclajes, siendo $1=$ Totalmente en desacuerdo y $5=$ Totalmente de acuerdo.

Variables socio-demográficas: para recolectar este tipo de información se utilizó un cuestionario ad-hoc que indagaba las siguientes variables: edad y género.

\section{RESULTADOS ALCANZADOS}

En primer lugar, se analizaron las actitudes hacia la inclusión educativa de la población magrebí. En la Tabla 1, se presentan los estadísticos descriptivos: puntuaciones medias $(M)$, desviaciones típicas $(D T)$, asimetría $(S)$ y curtosis $(K)$. 
Tabla 1. Análisis descriptivo sobre las actitudes hacia la inclusión educativa de la población magrebí.

\begin{tabular}{lllll}
\hline Actitudes hacia la inclusión educativa $(\alpha=.88)$ & $M$ & $D T$ & $S$ & $K$ \\
\hline $\begin{array}{l}\text { 1. Las universidades deberían garantizar el acceso } \\
\text { de estudiantes inmigrantes magrebíes. }\end{array}$ & 3,66 & 1,44 &,- 66 &,- 88 \\
& & & & \\
2. El gobierno debe garantizar que los hijos e hijas \\
de inmigrantes magrebíes reciban la educación \\
necesaria.
\end{tabular}

Como se observa, en general la población española muestra mayor acuerdo en relación a los items 3, 4 y 5, relacionados con la promoción de políticas públicas que promuevan la inclusión y el reconocimiento de la población magrebí. Sin embargo, en los items 1 y 2 las personas autóctonas muestran un menor acuerdo en relación a la inclusión de un alumnado magrebí, tanto a las escuelas como a las universidades españolas. Por otro lado, siguiendo a Hair et al. (2006), todos los items mostraron niveles adecuados en los índices de asimetría y curtosis $(+2 /-2)$.

En segundo lugar, se analizaron las diferencias en base al género para las variables estudiadas. Por un lado, no se han encontrado diferencias significativas en cuanto al prejuicio sutil. Por otro lado, como se muestra en la Figura 1, se analizaron las diferencias en las actitudes hacia la inclusión educativa del colectivo magrebí en función de los niveles de prejuicio sutil. 
Figura 1. Diferencia de medias en las actitudes hacia la inclusión educativa del colectivo magrebí según el género.

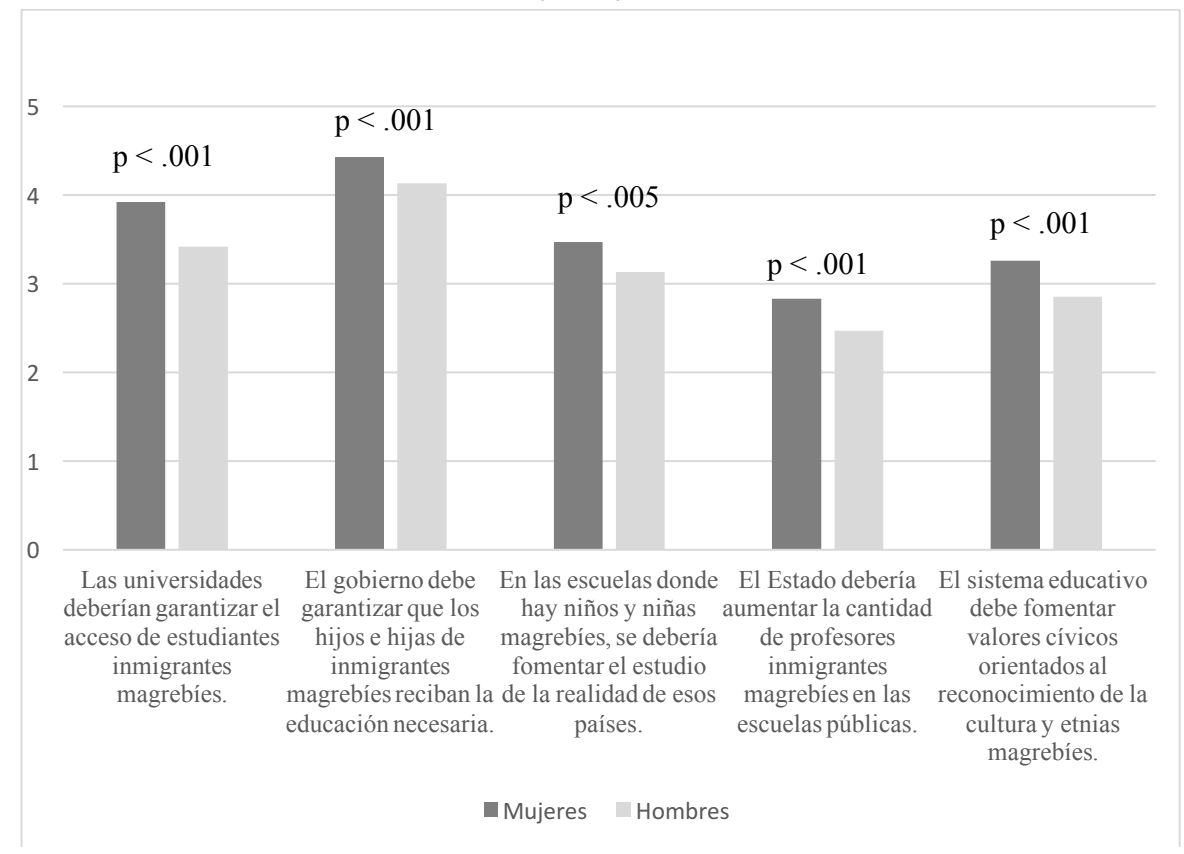

En este sentido, se indagó en las diferencias según el género en cuanto a las actitudes hacia la inclusión en la educación de la población magrebí y el prejuicio sutil hacia este mismo colectivo migrante. Las personas que se identificaron como mujeres $(M=3,92 ; D T=1,32)$ mostraron estar de acuerdo en mayor medida que los que se identificaron como hombres ( $M=3,42 ; D T=1,51)$ en que las universidades deberían garantizar el acceso de estudiantes inmigrantes magrebíes $(t(481)=3,857, p<.01)$. A su vez, pero en menor medida, las mujeres $(M=3,27$; $D T=1,53)$ mostraron una actitud más positiva que los hombres $(M=2,85$; $D T=1,54)$ en cuanto a que el sistema educativo debe fomentar valores cívicos orientados al reconocimiento de la cultura y etnias magrebíes ( $t(481)=$ $2,961, p<.01)$, en que el Estado debería aumentar la cantidad de profesores inmigrantes magrebíes en las escuelas públicas ( $\left.t_{(481)}=2,794, p<.01\right)$ y a que el gobierno debe garantizar que los hijos de inmigrantes magrebíes reciban la educación necesaria $(t(481)=2,745 p<.01)$. En última instancia, se encontró que las mujeres también tienen más actitudes positivas que los hombres en relación a que en las escuelas donde hay niños y niñas magrebíes, se debería fomentar el estudio de la realidad de esos países ( $t(481)=2,547, p<.05)$.

Por otro lado, en cuanto al prejuicio sutil de las personas participantes del estudio, no se han encontrado diferencias significativas en relación al género.

Por último, se evidenciaron correlaciones significativas $(p<, 01)$ negativas entre los niveles de prejuicio sutil y las actitudes hacia la inclusión educativa del profesorado y el alumnado magrebí (Tabla 2). 
Tabla 2. Correlaciones entre el prejuicio sutil y las actitudes hacia la inclusión de la población magrebí en el ámbito educativo.

1. Prejuicio sutil a migrantes

2. Las universidades deberían garantizar el acceso de estudiantes inmigrantes magrebíes.

3. El gobierno debe garantizar que los hijos de inmigrantes magrebíes reciban la educación necesaria.

4. En las escuelas donde hay niños y niñas magrebíes, se debería fomentar el estudio de la realidad de esos países.

\section{El Estado debería} aumentar la cantidad de profesores inmigrantes magrebíes en las escuelas públicas.

6. El sistema educativo debe fomentar valores cívicos orientados al reconocimiento de la cultura y etnias magrebíes.
.75

$-, 41 * * \quad-, 40 * * \quad-, 42 * * \quad-, 51 * * \quad-, 55 * *$

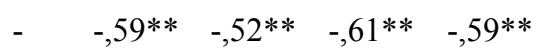

**. $p<.01 ; * . p<.05$. Nota. Alfa de Cronbach en la diagonal.

En este sentido, se encontraron relaciones con mayor fuerza entre el prejuicio sutil y las actitudes hacia la inclusión educativa de la población magrebí, en los siguientes items: el sistema educativo debe fomentar valores cívicos orientados al reconocimiento de la cultura y etnias magrebíes $(r=-, 55 ; p<, 001)$; el Estado debería aumentar la cantidad de profesores/as inmigrantes magrebíes en las escuelas públicas $(r=-, 51 ; p<, 001)$. Por otro lado, se encontraron correlaciones negativas, pero con menor fuerza en las actitudes que expresan que en las escuelas donde hay niños y niñas magrebies, se debería fomentar el estudio de la realidad de esos países ( $r$ $=-, 42 ; p<, 001)$, que las universidades deberían garantizar el acceso de estudiantes inmigrantes magrebíes ( $r=$ $-, 41 ; p<, 001)$ y en que el gobierno debe garantizar que los hijos e hijas de inmigrantes reciban la educación necesaria $(r=-, 40 ; p<, 001)$.

\section{DISCUSIÓN}

Los niveles de prejuicio sutil encontrados hacia la población migrante general de origen magrebí, se encuentran relacionados con las actitudes hacia la inclusión del profesorado y alumnado de la misma procedencia. Para erradicar los niveles de prejuicio hacia la diversidad cultural, así como las diferentes formas de discriminación que suponen, es necesario continuar analizando las diferentes variables implicadas. En este sentido, el ámbito 
educativo supone un lugar de intercambio e interacción que, en ocasiones, forma parte de la reproducción de desigualdades sociales, pero que también puede suponer un contexto de transformación social (Freire, 2005). Analizar las diferentes representaciones y actitudes sobre este ámbito es clave de cara a que los espacios educativos puedan aspirar a tener dicho carácter transformador. Además, se hace necesario contar con la presencia cada vez mayor de profesorado y alumnado capaz de representar la riqueza sociocultural existente en las sociedades actuales y futuras. Así, una medida que favorecería dicho enfoque es incorporar a profesorado con raíz cultural magrebí en los centros educativos en una proporción similar a la que existe en nuestra sociedad. En este sentido, sería necesario conocer e identificar la presencia de este profesorado en los distintos niveles educativos. Para todo ello será imprescindible sumar la presencia de políticas de justicia económica que garanticen medidas estructurales y una distribución justa de los recursos (materiales y culturales) en las aulas (Rawls, 1971) a estos colectivos en situación desfavorecida. Así, de cara a futuras líneas de investigación se sugiere la evaluación del prejuicio también en su forma manifiesta, así como de otras variables psicosociales relacionadas con la comprensión de la Educación como una herramienta válida para una convivencia intercultural más justa.

\section{CONCLUSIONES}

Los resultados permiten inferir que cuando existen altos niveles de prejuicio sutil hacia las personas migrantes de origen magrebí, las personas participantes también poseen actitudes más desfavorables hacia la inclusión educativa del profesorado y alumnado con este mismo origen. De esta forma, el ámbito educativo forma parte de las diversas manifestaciones de discriminación y exclusión que las personas establecen en base a sus niveles de prejuicio hacia un grupo social. Además, en coherencia con estudios anteriores (Mercado y Lira, 2021) el género es una variable a considerar en el análisis del prejuicio y dichas actitudes excluyentes, siendo las mujeres quienes menos niveles de prejuicio muestran. Así, se hace necesario considerar dicha variable, así como otras de carácter psicosocial, en el estudio del prejuicio y sus posibles consecuencias en la forma de entender la participación que deben poseer las personas migrantes en ámbito educativo.

En los últimos años, han sido numerosas las crisis (socioeconómicas, sanitarias, bélicas, entre otras) que han obligado a millones de personas en todo el mundo a iniciar un proceso migratorio. Las sociedades modernas y posmodernas se encuentran en un proceso continuo de transformación, con realidades cada vez más multiculturales. Dicha diversidad representa una fuente de riqueza sociocultural de un gran valor, que también requiere de la generación de espacios de convivencia basados en la perspectiva intercultural (Velasco Arroyo, 2020). Por ello, se sugiere la compatibilización de espacios de intervención socioeducativos basados en la educación inclusiva e intercultural (López Belmonte et al., 2018), centrados en la promoción de comunidades educativas que representen cada vez más la diversidad cultural presente en la sociedad, reduciendo el prejuicio en cualquiera de sus manifestaciones.

\section{REFERENCIAS BIBLIOGRÁFICAS}

Albalá Genol, M. A., Etchezahar, E., y Maldonado Rico, A. F. (2021). Creencias sobre la inclusión y la justicia social en la educación: factores implicados. Revista Prisma Social, (33), 162-182.

Arroyo, M. J. G. (2017). La educación intercultural: un camino hacia la inclusión educativa. Revista de educación inclusiva, 6(2), 144-159.

Bustos Martínez, L., De Santiago Ortega, P. P., Martínez Miró, M. A., y Rengifo Hidalgo, M. S. (2019). Discursos de odio: una epidemia que se propaga en la red. Estado de la cuestión sobre el racismo y la xenofobia en las redes sociales. Revista Mediaciones Sociales, 18, 25-42.

Chambers, J. R., Schlenker, B. R., y Collisson, B. (2013). Ideology and prejudice: The role of value conflicts. Psychological Science, 24(2), 140-149.

Civalero, L., Alonso, D., y Brussino, S. (2019). Evaluación del prejuicio hacia inmigrantes: adaptación argentina de la escala de prejuicio sutil y manifiesto. Ciencias Psicológicas, 13(1), 119-133.

Freire, P. (2005). Pedagogía de la esperanza. Siglo XXI Editores. 
Gaertner, S. L., y Dovidio, J. F. (1986). The aversive form of racism. Academic Press.

Hair, J. F., Black, W. C., Babin, B. J., Anderson, R. E., y Tatham, R. (2006). Multivariate data analysis. Uppersaddle River.

Instituto Nacional de Estadística - INE (2020). Población extranjera por Nacionalidad, comunidades, Sexo y Año. INEbase. https://www.ine.es/jaxi/Tabla.htm?path=/t20/e245/p08/I0/\&file=02005.px\&L=0

Licata, L., Sanchez-Mazas, M., y Green, E. G. (2011). Identity, immigration, and prejudice in Europe: a recognition approach. En S. J. Schwartz, \& V. L. Vignoles (Eds.), Handbook of Identity Theory and Research (pp. 895916). Springer.

López Belmonte, J., Fuentes Cabrera, A., y Pozo Sánchez, S. (2018). Educación inclusive e intercultural al borde de la frontera: la escolarización del colectivo MENA. Revista de Pedagogía, 39(105), 173-196.

MECD (2021). Datos y cifras. Curso escolar 2020/2021. Secretaría General Técnica

Mercado, D. B., \& Lira, L. R. (2021). Percepción de amenaza y expresiones de prejuicio hacia la migración centroamericana indocumentada en tránsito por México. Andamios, Revista de Investigación Social, 18(45), 97120.

Pettigrew, T. F., y Meertens, R. W. (1995). Subtle and blatant prejudice in Western Europe. European journal of social psychology, 25(1), 57-75.

Rawls, J. (1971). A theory of justice. Oxford University Press.

Rueda, J. F., y Navas, M. (1996). Hacia una evaluación de las nuevas formas del prejuicio racial: las actitudes sutiles del racismo. Revista de psicología social, 11(2), 131-149.

Rueda, J. F., y Navas, M. (1996). Hacia una evaluación de las nuevas formas del prejuicio racial: las actitudes sutiles del racismo. Revista de Psicología Social, 11(2), 131-149.

Sapon-Shevin, M. (2013). La inclusión real: una perspectiva de justicia social. Revista de Investigación en Educación, 11 (3), 71-85.

Subdirección General de Estadística y Estudios. Datos y cifras. Ministerio de Educación y Formación Profesional. Velasco Arroyo, J. C. (2020). Migraciones internacionales y derechos humanos desde una perspectiva cosmopolita. Universidad Autónoma de México. 
